\title{
Variceal haemorrhage in hereditary haemorrhagic telangiectasia
}

\author{
P L ZENTLER-MUNRO, E R HOWARD, J KARANI AND ROGER WILLIAMS
}

From the Liver Unit, King's College Hospital, London

\begin{abstract}
SUMmARY Hepatic in involvement in hereditary haemorrhagic telangiectasia can lead to cirrhosis and occasionally to portal hypertension and variceal haemorrhage. The ultrasonographic, arteriographic and histological findings are described in a patient with this complication. Hepatic artery embolisation proved unsuccessful in arresting repeated haemorrhage which was eventually controlled by hepatic artery ligation. Porto-systemic venous shunting, an apparently logical approach to management, would probably have aggravated the problem.
\end{abstract}

Hepatic involvement in hereditary haemorrhagic telangiectasia (HHT) is rare, but has been described in various forms including hepatic vascular abnormalities, fibrosis and cirrhosis. Martini, in the last major review,' pointed out that the liver could be involved by telangiectasis, fibrosis, or both. In the absence of fibrosis, vascular abnormalities shown by angiography have included arterioportal and arteriovenous fistulae, hepatic artery aneurysms, and cavernous haemangiomas. Martini has also described a specific form of macronodular cirrhosis, particularly marked in the subcapsular area and comprising broad irregular fibrous septae containing telangiectasiae of varying size. The connective tissue contained epithelial sprouts similar to those seen in bile duct proliferation, and blood filled cavities lined by intimal endothelium only. The arterioles were abnormal with intimal thickening and microthrombi; venules contained hypertrophied longitudinal and circular muscle. 'In the cases included in Martini's review, portal hypertension and variceal bleeding seemed relatively infrequent. We discuss here the management of a man with extensive HHT and recurrent variceal haemorrhage, who had been referred for consideration of a portosystemic venous shunt operation.

Address for correspondence: Dr P Zentler-Munro. Raigmore Hospital. Inverness IV2 3UJ.

Accepted for publication 7 February 1989.
CASE HISTORY

The patient, a 51 year old Greek naval officer, presented in 1977 at the age of 42 with anaemia, frequent epistaxis, and rectal bleeding; numerous skin telangiectases, hepatomegaly and an hepatic bruit were noted at that time. Mesenteric angiography in 1979 showed telangiectases throughout the intestine and particularly in the colon. The hepatic artery was embolised unsuccessfully with Gelfoam, but further attempts in 1983 (Ivalon particles) and 1984 (Teflon coils) eventually achieved a reduction in the bruit and associated hyperdynamic circulation. In 1986 he developed acites for the first time, and was found to have oesophageal varices on oesophagoscopy which were treated by sclerotherapy. A few months later he suffered the first of several major variceal haemorrhages. He was otherwise well and had drunk very little alcohol all his life.

The patient's family history was extensive: his mother, four maternal uncles and one brother suffered from HHT and cirrhosis. None had ever drunk significant quantities of alcohol. His mother, one uncle and his brother had died of variceal haemorrhage.

Examination revealed many telangiectases over the lips, tongue, palate, ears, and fingers; there were many spider naevi over the upper trunk and palmar erythema. The liver was palpable $8 \mathrm{~cm}$ below the costal margin and the spleen was just palpable; acites was present and there was a loud hepatic bruit. 


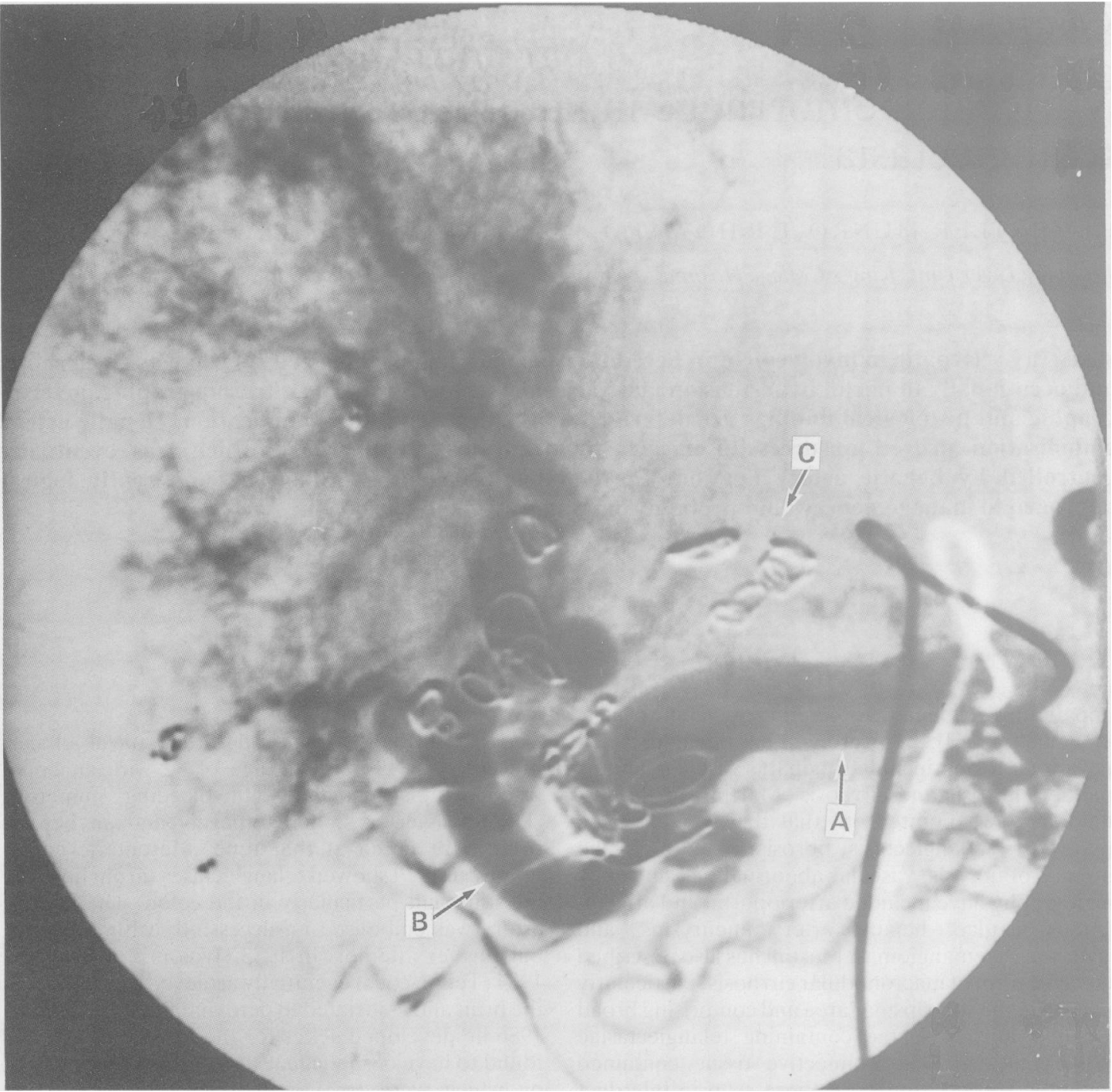

Fig. 1 Hepatic arteriogram (early) showing enlarged common hepatic (A) and right hepatic (B) arteries contuining se'veral coils, with further coils in site of occluded lefi hepatic artery (C).

The heart was enlarged with a left ventricular heave, ejection systolic murmur and hyperdynamic peripheral circulation; the jugular venous pulse was normal.

Investigations showed a mild hypochromic microcytic anaemia ( $\mathrm{Hb} 9.7 \mathrm{~g} / \mathrm{dl}$ ), abnormal alkaline phosphatase (167 IU/l) and gamma glutamyl transpeptidase (173 IU/l), but normal aspartate transaminase (30 IU/l); albumin was normal (41 g/l) but prothrombin time raised $(17 \mathrm{sec}$, control $14 \mathrm{sec})$. Abdominal ultrasonography showed an enlarged liver and spleen with homogeneous parenchyma. The hepatic artery was enlarged with Teflon coils oscillating within the arterial lumen. Doppler studies showed turbulent flow within the dilated hepatic artery. There was reverse (hepatofugal) flow within the right branch of the portal vein and main extrahepatic portal vein with forward (hepatopetal) flow within the left branches.

Arteriography confirmed the enlarged hepatic artery: occlusive coils were present in the common hepatic artery and right hepatic artery, but major 


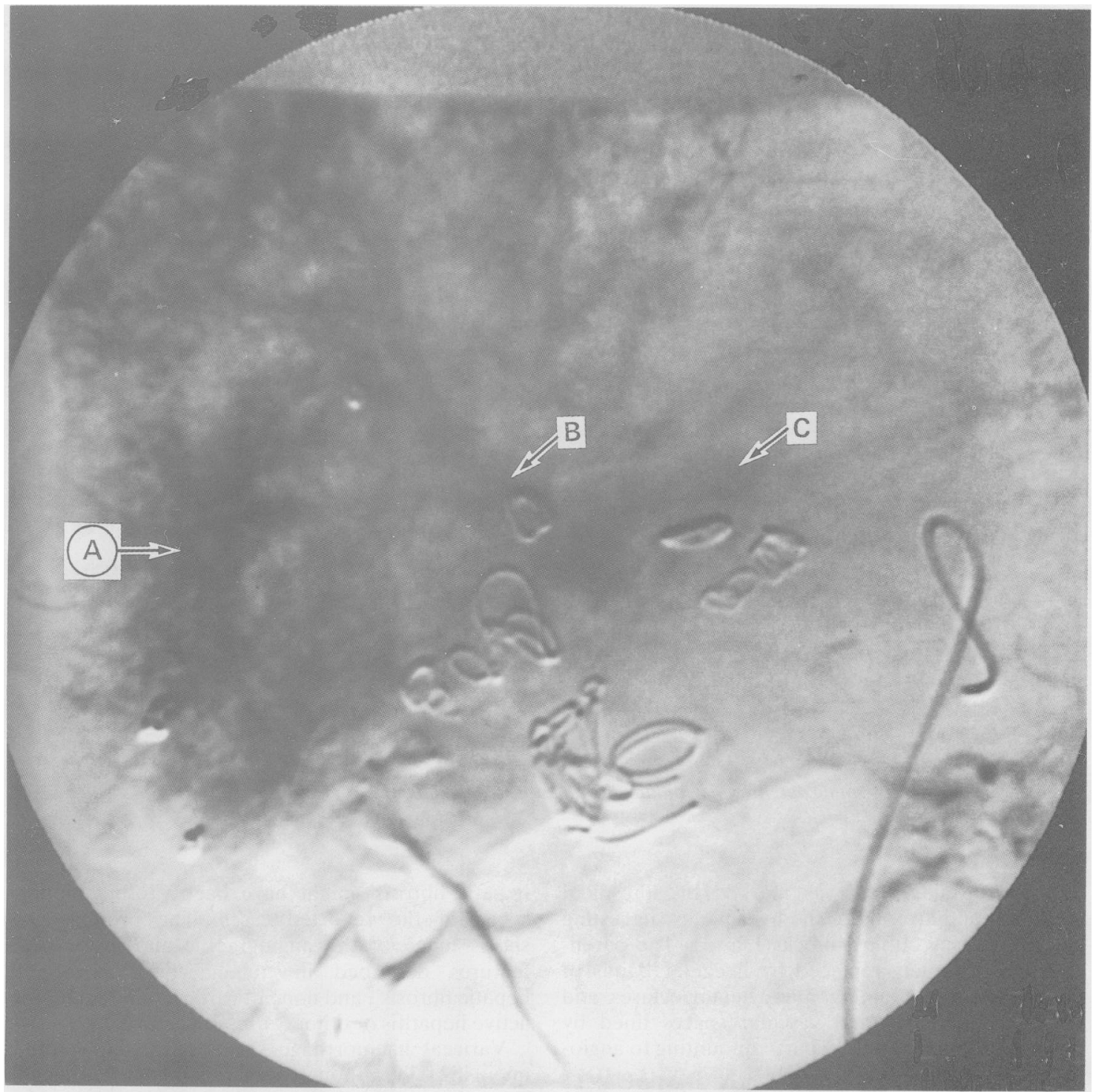

Fig. 2 Hepatic arteriogram (late) showing arterioportal venous fistulae (A) and retrograde filling of right branch of portal vein $(\mathrm{B})$ and extrahepatic portal vein $(\mathrm{C})$.

arterial flow continued with numerous arterioportal venous fistulac (Fig. 1). The left hepatic artery had been successfuly occluded and no fistulae were demonstrated. There was, consequently, retrograde filling of the right branch of the portal vein which filled the left branch of the portal vein and the extrahepatic portal vein (Fig. 2).

A portosystemic shunt operation was clearly contraindicated as it would have increased flow through the arterioportal fistulae and portal venous system.
Open hepatic artery ligation was therefore performed (Mr E R Howard) without any postoperative problem. The bruit disappeared immediately and on the fourth day Doppler ultrasonography showed normal low volume hepatopetal portal venous blood flow. On the 11th day direct splenic venography showed a normal portal venous system with forward flow and no oesophageal variceal circulation.

A wedge liver biopsy taken at operation showed advanced micronodular cirrhosis with regeneration. 


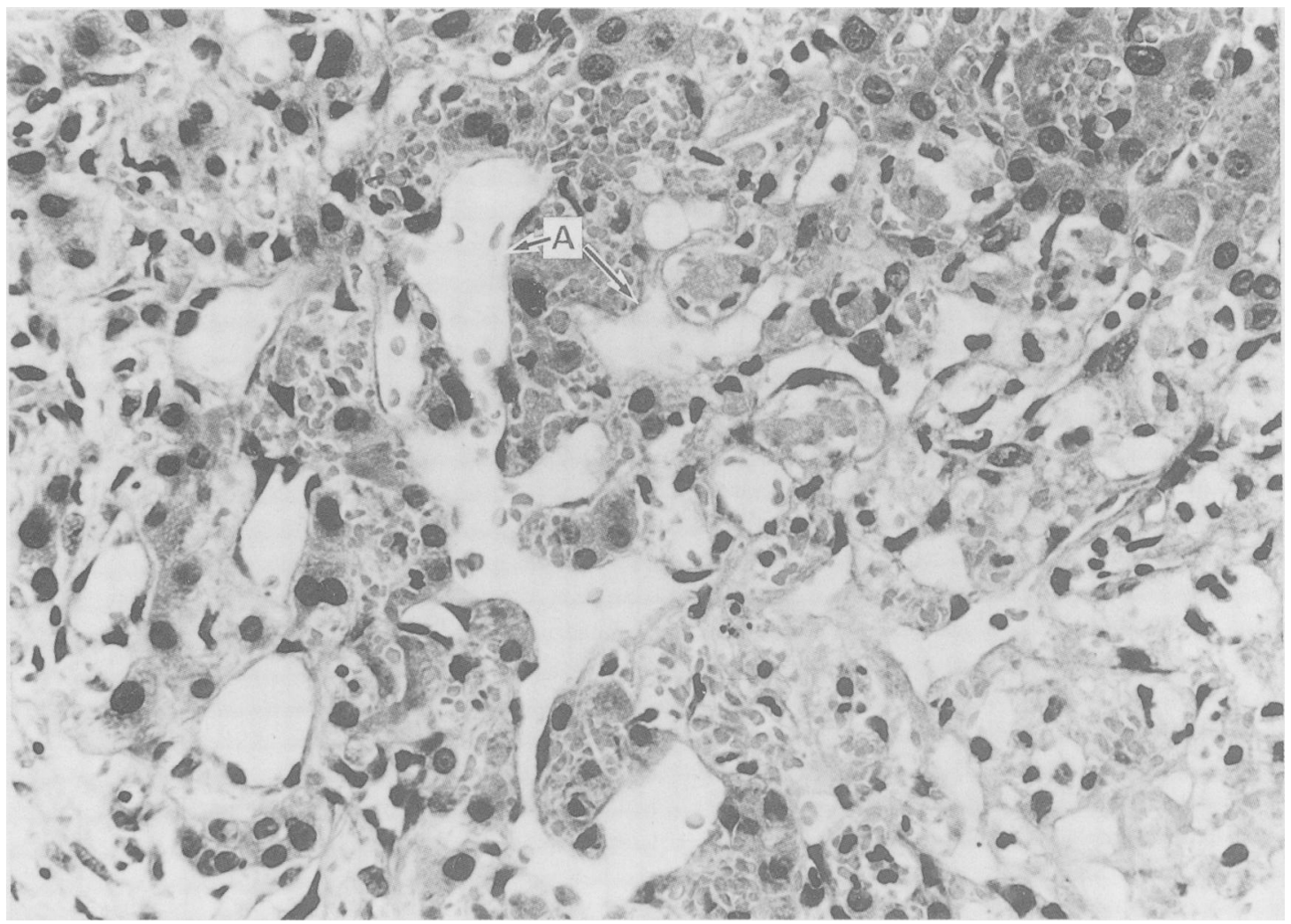

Fig. 3 Portion of regenerating parenchymal nodule showing sinusoidal dilatation (A) with consecutive atrophy and loss of intervening hepatocytes. $H \& E$.

The subcapsular area was marked by sinusoidal dilatation and atrophy of the liver plates suggesting chronic venous outflow block (Fig. 3). The parenchyma was largely replaced by irregular bands of fibrous tissue containing many telangiectases and irregular interconnected vascular spaces lined by normal endothelial cells in parts amounting to angiomatous transformation (Fig. 4).

Six months later the patient returned for review. He remained well with no further variceal hacmorrhage, and regression of the varices on oesophagoscopy. Examination by Doppler ultrasonography again showed normal low velocity forward portal venous flow.

\section{Discussion}

Cooney et $a l^{2}$ pointed out that few reports of HHT distinguished between fibrosis and cirrhosis, or excluded other causes of cirrhosis - particularly those due to chronic active hepatitis or cardiac failure. They suggested that true 'telangiectasia associated hepatic fibrosis' might have been present in only seven of the reported cases. The present case shows many of the angiographic and histological features described in telangiectasia associated hepatic fibrosis", and none of cardiac failure, chronic active hepatitis or other causes of cirrhosis.

Variceal haemorrhage seems surprisingly infrequent in HHT, although perhaps it is not often sought by endoscopy in patients who are assumed to be bleeding from telangiectases in the gut. Rewane reported angiographic abnormalities in a patient who subsequently died of variceal hacmorrhage. He described hepatofugal flow in the right portal vein and failure of hepatic venous filling, as in the present case. Autopsy showed telangiectatic cirrhosis with 'angiomatous' fibrous bands; hepatic venous obstruction was attributed to fibrosis and regenerative nodules. Radtke et al ${ }^{+}$reported two patients with hepatic arteriovenous rather than arterioportal fistulae leading to heart failure. Hepatic artery ligation was performed successfully, but Danchin et $a^{\prime}$ suggested that the improvement might prove short 


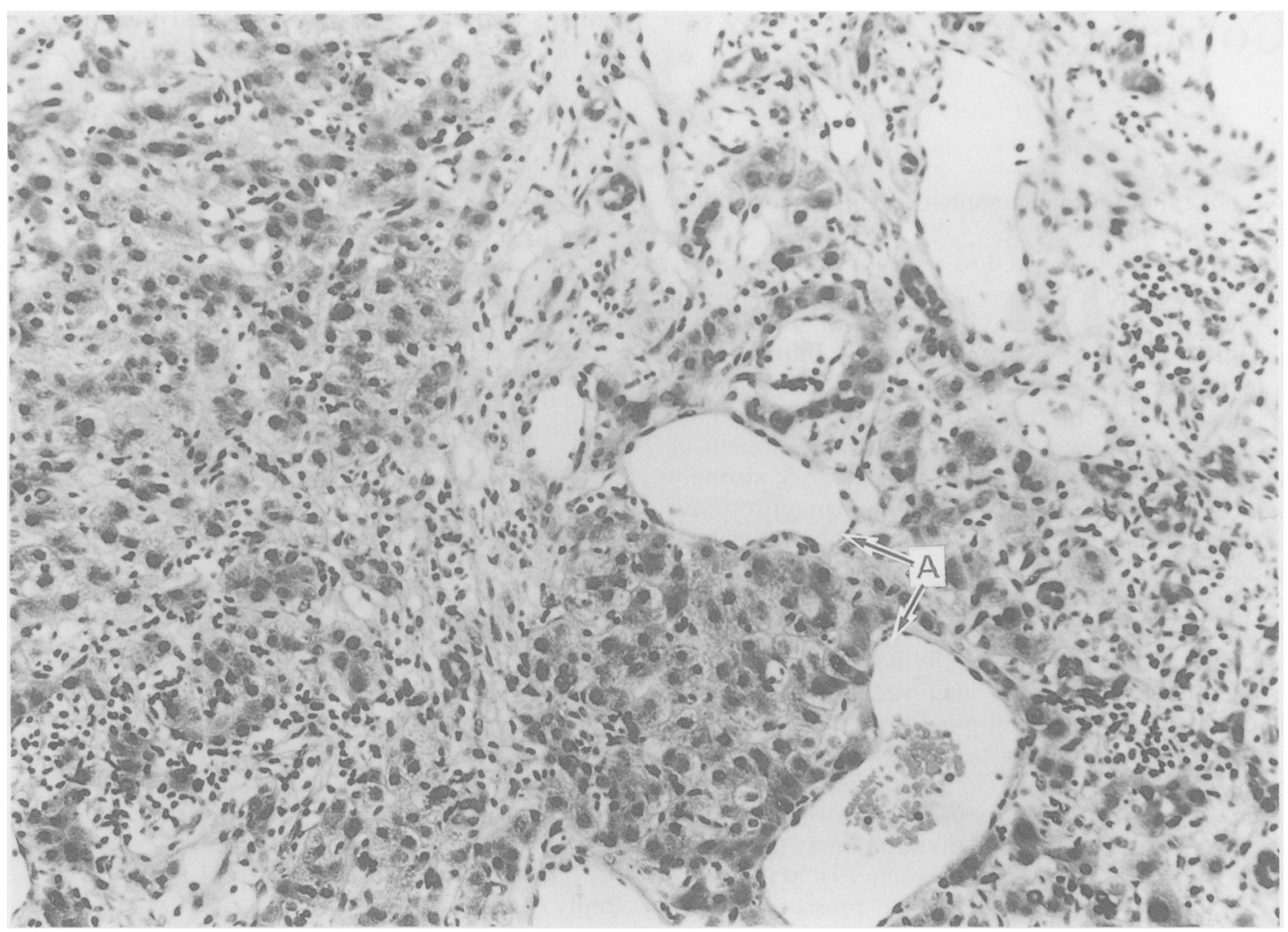

Fig. 4 Fibrous areas within parenchyma containing many thin-walled and dilated vascular spaces (A). $H \& E$.

lived because of the development of a collateral circulation.

The arteriographic and histological findings reported by Martini' and Cooney' suggest that portal hypertension is usually a consequence of arterioportal fistulae rather than cirrhosis, and this might suggest that variceal sclerotherapy is unlikely to prove successful because of the very high flow in the varices. The usually well preserved liver function and absence of encephalopathy, on the other hand, might suggest that portosystemic shunting would prove ideal. The ultrasonographic and angiographic studies in our case, however, indicate that shunting would almost certainly have aggravated portal venous blood flow and collateral circulation. Hepatic artery ligation seems a more rational approach, and has produced an excellent result in this case.

\section{References}

1 Martini (jA. The liver in hereditary haemorrhagic telangiectasiase: an inborn error of vascular structure with multiple manifestations: a reappraisal. (jut 1978: 19: $531-7$.

2 Cooncy T, Sweeney EC, Coll R, Greally M. 'Pseudocirrhosis' in hereditary hacmorrhagic telangiectasiac. $J$ Clin Puthol 1977; 30: 1134-41.

3 Rewane 1. Hereditary hacmorrhagic telangiectasiac (Osler`s disease) with special reference to angiographic findings in liver cirrhosis. Br J Radiol 1983; 56: 207-9.

4 Radtke WE. Smith HC. Fulton RE. Adson MA. Misdiagnosis of atrial septal defect in patients with hereditary telangiectasiae (Osler-Weber-Rendu disease) and hepatic arteriovenous fistulas. Am Heart J 1978: 95: 235-42.

5 Danchin N. Thisse JY. Niemann JL. Faivre G. OslerWeber-Rendu discase with multiple intrahepatic arteriovenous fistulas. Am Heart J 1983; 105: 856-9. 